\title{
Seminal Cell Free DNA Concentration Levels Discriminate Between Prostate Cancer and Benign Prostatic Hyperplasia
}

\author{
GIOVANNI PONTI ${ }^{1}$, MONIA MACCAFERRI $^{1}$, SALVATORE MICALI $^{2}$, MARCO MANFREDINI $^{3}$, \\ RICCARDO MILANDRI ${ }^{2}$, GIAMPAOLO BIANCHI ${ }^{2}$, GIOVANNI PELLACANI ${ }^{3}$, SHANIKO KALECI ${ }^{3}$, \\ JOHANNA CHESTER ${ }^{3}$, ANDREA CONTI $^{3}$, CHIARA DEL PRETE $^{2}$ and ALDO TOMASI ${ }^{1}$ \\ ${ }^{1}$ Division of Clinical Pathology, Department of Surgical, Medical, \\ Dental and Morphological Sciences with Interest in Transplant, \\ ${ }^{2}$ Division of Urology, Department of Surgical, Medical, \\ Dental and Morphological Sciences with Interest in Transplant, \\ ${ }^{3}$ Dermatology Unit, Department of Surgical, Medical, Dental and Morphological \\ Sciences with Interest in Transplant, University of Modena and Reggio Emilia, Modena, Italy
}

\begin{abstract}
Background/Aim: Seminal plasma cfDNA (scfDNA) was recently proposed as a novel PCa biomarker. Our aim was to evaluate whether scfDNA could discriminate $P C$ a from benign prostate hyperplasia $(B P H)$ patients. Patients and Methods: A cohort of 43 patients (18 and 25 pathology proven $\mathrm{PC}$ a and BPH patients), and 13 healthy age-matched control subjects were enrolled. scfDNA quantification was performed. Data were analyzed through ANOVA testing. Results: Average scfDNA concentrations were $1,407.83 \mathrm{ng} / \mu \mathrm{l}, 128.13 \mathrm{ng} / \mu \mathrm{l}$ and $78.09 \mathrm{ng} / \mu \mathrm{l}$ for PCa patients, BPH patients and healthy subjects, respectively. Statistical analysis showed a significant difference among the groups, allowing for distinction of patients with optimal accuracy. A cut-off level of $450 \mathrm{ng} / \mu \mathrm{l}$ scfDNA was identified for the differentiation of $\mathrm{PCa}$ and $\mathrm{BPH}$ patients. Conclusion: scfDNA concentrations are significantly different between $P C a$ patients and BPH patients. scfDNA is a promising biomarker with several applications in PCa diagnosis, screening programs and therapeutic monitoring.
\end{abstract}

Prostate cancer (PCa) is the most common malignant disease and the second most frequent cause of cancer-

Correspondence to: Giovanni Ponti, Division of Clinical Pathology, Department of Surgical, Medical, Dental and Morphological Sciences with Interest in Transplant, University of Modena e Reggio Emilia, Via del Pozzo n 71, 41124, Modena, Italy. Tel: +39 0594224748, Fax: +39 0594224271, e-mail: giovanni.ponti@unimore.it

Key Words: Seminal plasma cfDNA, spermal cfDNA, prostate cancer, urological biomarkers, fluorimetry, prostate cancer screening, liquid biopsy. related mortality amongst men worldwide (1). The advent of liquid biopsy techniques and the isolation of circulating tumor DNA (ctDNA) from blood or seminal fluid, may represent a major step forward in $\mathrm{PCa}$ diagnosis and management compared to traditional diagnostic techniques involving prostate-specific antigen (PSA), digital rectal examination (DRE), transrectal ultrasound (TRUS) and biopsy (2-5).

The use of PSA as a serum marker has revolutionized PCa diagnosis. However, this antigen is not cancer-specific and may be elevated in other non-malignant conditions, such as benign prostatic hyperplasia (BPH) and prostatitis $(6,7)$. Several new biological markers such as BRCA2 $(8$, 9), TMPRSS2-Erg fusion and PCA3 (10) and four-kallikrein panel (11) have been shown to add sensitivity and specificity to PSA, thereby decreasing the incidence of $\mathrm{PCa}$ over-diagnosis. Current evidence regarding biomarkers is too limited for the development of recommendations. Further, a population-based survival benefit has not yet been demonstrated.

Within the setting of liquid biopsy, circulating free (cfDNA) and ctDNA quantification and sequencing allow the collection of important data regarding cancer diagnosis and characterization, patient prognosis and management in order to determine therapeutic strategies and the subsequent follow-up $(12,13)$. Higher blood plasma cfDNA levels in PCa patients with respect to healthy subjects have been reported (3-5), as well as significant differences in cfDNA levels and integrity between PCa and BPH patients $(14,15)$. Previous studies have identified urine cell-free DNA (UCF-DNA) integrity as a potential marker for early diagnosis of non-invasive prostate cancers, even though there are no large cohort studies supporting this hypothesis $(16,17)$. 
Table I. Clinical, pathological features of prostate cancer patients, BPH patients and healthy individuals.

\begin{tabular}{|c|c|c|c|c|c|c|c|c|c|c|}
\hline $\begin{array}{l}\text { PC } \\
\text { Patients }\end{array}$ & Age & $\begin{array}{l}\text { PSA } \\
(\mathrm{ng} / \mathrm{ml})\end{array}$ & $\begin{array}{c}\text { Gleason } \\
\text { Score }\end{array}$ & $\begin{array}{c}\text { Qubit ssDna } \\
(\mathrm{ng} / \mu \mathrm{l})\end{array}$ & $\begin{array}{c}\mathrm{BPH} \\
\text { Patients }\end{array}$ & Age & $\begin{array}{c}\text { PSA } \\
(\mathrm{ng} / \mathrm{ml})\end{array}$ & $\begin{array}{l}\text { Qubit ssDna } \\
(\mathrm{ng} / \mu \mathrm{l})\end{array}$ & Healthy & $\begin{array}{c}\text { Qubit ssDna } \\
(\mathrm{ng} / \mu \mathrm{l})\end{array}$ \\
\hline 1 & 67 & 8.7 & $7(4+3)$ & $1,635.00$ & 1 & 67 & 4.49 & 215.40 & 1 & 62.50 \\
\hline 2 & 61 & 7.05 & $6(3+3)$ & $2,100.00$ & 2 & 55 & 4.9 & 76.10 & 2 & 52.90 \\
\hline 3 & 65 & 6.2 & $7(3+4)$ & 978.00 & 3 & 52 & 7.52 & 110.00 & 3 & 43.20 \\
\hline 4 & 74 & 4.02 & $6(3+3)$ & 780.00 & 4 & 66 & 9.5 & 64.00 & 4 & 124.60 \\
\hline 5 & 76 & 4.37 & $7(4+3)$ & $2,340.00$ & 5 & 64 & 7.37 & 278.00 & 5 & 112.30 \\
\hline 6 & 63 & 4.73 & $7(3+4)$ & 879.00 & 6 & 63 & 4.13 & 74.00 & 6 & 67.90 \\
\hline 7 & 69 & 7.02 & $7(4+3)$ & 980.00 & 7 & 67 & 6 & 67.80 & 7 & 85.40 \\
\hline 8 & 58 & 14 & $7(3+4)$ & 789.00 & 8 & 51 & 5.77 & 276.00 & 8 & 56.73 \\
\hline 9 & 64 & 6.06 & $6(3+3)$ & 856.00 & 9 & 60 & 3.7 & 76.65 & 9 & 45.67 \\
\hline 10 & 66 & 12.12 & $7(4+3)$ & $2,280.00$ & 10 & 57 & 4.14 & 89.00 & 10 & 87.54 \\
\hline 11 & 71 & 6.14 & $8(5+3)$ & 975.00 & 11 & 65 & 4.55 & 135.00 & 11 & 115.24 \\
\hline 12 & 74 & 5.37 & $6(3+3)$ & 871.00 & 12 & 61 & 4.42 & 63.50 & 12 & 62.56 \\
\hline 13 & 71 & 12.66 & $6(3+3)$ & $1,120.00$ & 13 & 58 & 4.02 & 93.70 & 13 & 98.67 \\
\hline 14 & 65 & 7.15 & $7(3+4)$ & $1,482.00$ & 14 & 60 & 4.52 & 75.40 & & \\
\hline 15 & 59 & 6.89 & $6(3+3)$ & $2,123.00$ & 15 & 63 & 3.89 & 218.00 & & \\
\hline 16 & 56 & 18 & $7(3+4)$ & $2,230.00$ & 16 & 61 & 4.2 & 150.00 & & \\
\hline 17 & 68 & 6.5 & $6(3+3)$ & $1,167.00$ & 17 & 67 & 4.88 & 69.70 & & \\
\hline \multirow[t]{8}{*}{18} & 70 & 7.4 & $6(3+3)$ & $1,756.00$ & 18 & 67 & 3 & 76.50 & & \\
\hline & & & & & 19 & 55 & 4.25 & 115.30 & & \\
\hline & & & & & 20 & 65 & 7.12 & 134.20 & & \\
\hline & & & & & 21 & 68 & 5.75 & 87.90 & & \\
\hline & & & & & 22 & 70 & 4.2 & 232.80 & & \\
\hline & & & & & 23 & 64 & 3.4 & 89.70 & & \\
\hline & & & & & 24 & 68 & 5.6 & 145.20 & & \\
\hline & & & & & 25 & 68 & 6.3 & 189.40 & & \\
\hline Mean & 67 & 8.02 & & $1,407.83$ & & 62.48 & 5.10 & 128.13 & & 78.09 \\
\hline SD & 5.6 & 3.07 & & 585.11 & & 4.29 & 1.28 & 68.00 & & 27.73 \\
\hline
\end{tabular}

So far, no research has been dedicated to cfDNA assessment in seminal plasma of PCa and BPH patients. Seminal fluid, which also originates from prostatic secretions, can be a privileged source of biomarkers for PCa diagnosis. cfDNA has been detected in human semen at higher concentrations compared to other biological fluids $(3,4,18)$. Seminal cfDNA is a potentially strong biomarker with several oncologic applications for PCa risk stratification, diagnosis and therapeutic monitoring. Recently, we reported that the fluorometric quantification and the electrophoretic characterization of cfDNA in seminal plasma allow the discrimination between PCa patients and age-matched healthy individuals, demonstrating significantly higher concentrations of cfDNA in the former group, with a distinct electrophoretic pattern (4). In these studies, we demonstrated that the fluorometric approach is an easy and reliable method to quantify cfDNA concentration in PCa patients. Furthermore, the electrophoresis technique is an efficient and cost-effective method, which is particularly suitable for the quantification of DNA size distribution patterns and integrity (13). The aim of this study was to evaluate fluorimetric quantification of seminal cfDNA as a biomarker for discrimination of PCa from $\mathrm{BPH}$ patients and healthy individuals.

\section{Materials and Methods}

Ethics statement. The study was approved by the ethics committee of the University of Modena and Reggio Emilia and informed consent was obtained from each recruited subject.

This study was conducted according to the principles of the Helsinki Declaration of 1975, amended in 1996 (http:// www.wma.net/e/humanrights/policy_meetings.htm).

Sample collection. The Urology Department of the University of Modena and Reggio Emilia enrolled patients with histopathologically proven prostate cancer, and BPH. Healthy individuals were enrolled as a control group. Patient eligibility criteria included age between 50 and 75 years, seminal fluid samples collected at least one month after prostate biopsy, availability of clinical data and PSA levels. Healthy individual's eligibility criteria included the absence of prostate pathology.

Patient seminal samples were collected prior to radical prostatectomy and/or chemotherapy or radiotherapy.

Extraction of cfDNA. As the samples ranged from 0.65 to $3.0 \mathrm{ml}$, a standardized sample volume of $0.65 \mathrm{ml}$ was analyzed for each sample collected. Samples processed within $2 \mathrm{~h}$ from collection were centrifuged at room temperature in 2 steps: $400 \mathrm{rcf}$ for $10 \mathrm{~min}$ and 16,000 ref, for $5 \mathrm{~min}$ (18). Seminal plasma samples were then stored at $-80^{\circ} \mathrm{C}$ awaiting cfDNA extraction. 
cfDNA was then extracted with the QIAamp Circulating Nucleic Acid Kit (Qiagen, Hilden, Germany) according to the manufacturer's instructions. Aliquots of $120 \mu \mathrm{l}$ cfDNA were cryopreserved at $-20^{\circ} \mathrm{C}$ until quantification.

Quantification of cfDNA. cfDNA concentration levels were measured using the Qubit ssDNA Assay Kit according to the manufacturer's instructions (Life Technologies, Carlsbad, CA, USA).

Statistical analysis. This article presents the statistical analysis of concentrations of cfDNA in seminal plasma in each group (PCa, $\mathrm{BPH}$ and healthy individuals).

MedCalc Statistical Software version 14.8.1 (MedCalc Software bvba, Ostend, Belgium; http://www.medcalc.org; 2014) was used to perform statistical analysis. The results are presented as the mean \pm standard deviation (SD) for each group. The mean effect of these groups was determined using analysis of variance (ANOVA). Box-and-whisker plot was plotted to compare the results.

Prior to the ANOVA test, Levene's Test for Equality of Variances was performed. If the Levene test was positive $(p<0.05)$, the variances in the different groups were different (the groups are not homogeneous) and logarithmic transformation for the data was applied. The program performs the calculations on the logarithms of the dependent variable, but the different means are backtransformed and reported as the geometric means.

ANOVA test, $\mathrm{F}$ statistic and associated $p$-value were calculated. If $p$-value was less than 0.05 , then the hypothesis is accepted that there is an influence of the qualitative factor on the dependent data, or that the means of at least two of the subgroups differ significantly.

If the ANOVA test was positive a post hoc test was performed (using Tukey-Kramer's method) for pairwise comparison of subgroups. A $p$-value $<0.05$ was considered significant.

\section{Results}

Patient population. Patients enrolled included $18 \mathrm{PCa}$ patients, $25 \mathrm{BPH}$ patients and 13 healthy individuals. The median age of the PCa patient population was 67 years (range $=58-76$ years), of the BPH patient population was 62.48 years (range $=51-70$ years) and of the healthy individuals was 66 years (range $=59-74$ years). Other clinical and pathological data are outlined in Table I.

Quantification of cfDNA. Median seminal cfDNA levels in $\mathrm{PCa}$ patients were higher than in $\mathrm{BPH}$ patients and healthy individuals $(p<0.001)$. Mean cfDNA level for PCa patients was $1,407.83 \mathrm{ng} / \mu \mathrm{l}$ (range $=780-2,340 \mathrm{ng} / \mu \mathrm{l})$, for $\mathrm{BPH}$ patients was $128.13 \mathrm{ng} / \mu \mathrm{l}$ (range $=63.5-278 \mathrm{ng} / \mu \mathrm{l})$ and for healthy individuals was $78.09 \mathrm{ng} / \mu \mathrm{l}$ (range=43.2-124.6 $\mathrm{ng} / \mu \mathrm{l})$, Table I. Seminal cfDNA concentrations were similar in $\mathrm{BPH}$ patients and healthy volunteers.

Comparison among groups. In this study, a significant difference was found between the concentrations of cfDNA in seminal plasma in $\mathrm{PCa}$ patients versus $\mathrm{BPH}$ patients

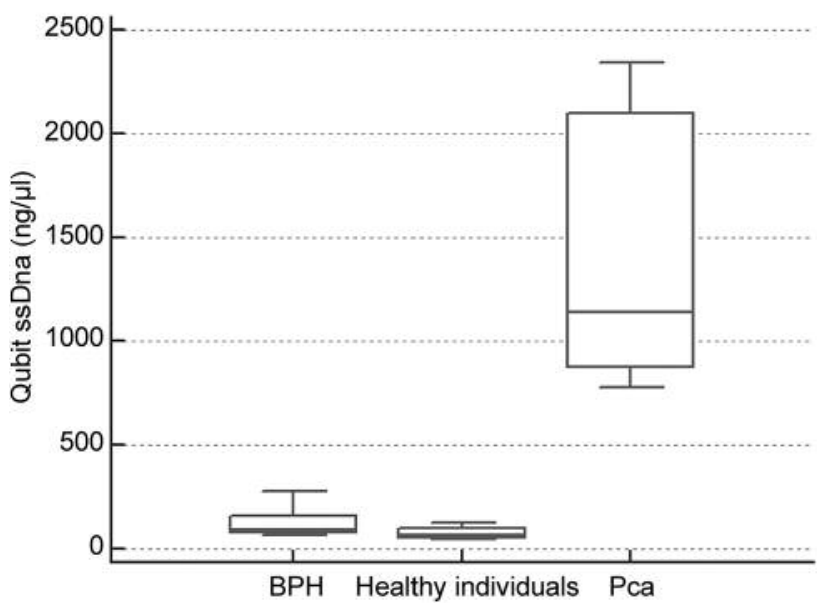

Figure 1. The Box-and-whisker plot displays a graphical statistical summary of a variable. In the basic Box-and-whisker plot, the central box represents the values from the lower to upper quartile (25 to 75 percentile). The middle line represents the median. The horizontal line extends from the minimum to the maximum value.

$(1,407.8 v s .128 .1$, ANOVA $p<0.001)$ and $\mathrm{PCa}$ patients versus healthy individuals (1,407.8 vs. 78.0, ANOVA $p<0.001)$. Box-and-whisker plot representing scfDNA values among groups are represented in Figure 1.

\section{Discussion}

In the present study scfDNA levels were evaluated as a potential clinical biomarker in early PCa diagnosis, using the total scfDNA amount in order to discriminate benign from malignant-prostate disease.

cfDNA can be found in biological fluids, such as plasma (2) urine (3), cerebral spinal fluid (4), pleural fluid (5), and saliva (6). ctDNA/cfDNA levels in blood samples seems to be dependent on tumour type. ctDNA was detected in over $75 \%$ of patients with advanced pancreatic, ovarian, colorectal, bladder, gastroesophageal, breast, melanoma, hepatocellular, and head and neck cancers. However, levels below $50 \%$ were found in primary brain tumours, kidney, prostate, and thyroid cancers (7). These findings may be due to the influence of other factors, such as inflammatory or autoimmune processes that increase non-tumour cfDNA fraction, thereby diluting the ctDNA concentration.

The identification, isolation and quantification of ctDNA from non-blood biological fluids, allows prompt diagnosis and a reliable genetic characterization of the cancer molecular signature. Thus, potential identification of ctDNA in nonblood fluids is of interest especially for cancers with low levels of ctDNA in blood samples. Some tumours are often in direct contact with specific non-blood bodily fluids, such as urine, saliva, cerebrospinal fluid, pleural effusion or 
seminal liquid, and release into these fluids a significant amount of ctDNA. These fluids therefore constitute a privileged source of ctDNA.

Concerning the relationship among cfDNA and ctDNA, a percentage of cfDNA in PCa patients was derived from noncancerous cells because of the induction of apoptosis by proapoptotic cytokines released from prostate cancer cells (8). Therefore, the quantification of total cfDNA level may be a more useful marker for cancer detection $(8,9)$. Today, it is possible to assess the precise amount of ctDNA through the identification of specific genetic mutations, present in cancer cells' nucleic acids. However, the precise differentiation between the amount of cfDNA derived from non-cancerous cells and the amount of ctDNA requires laboratory techniques such as qPCR or next generation sequencing (NGS) which are expensive and time consuming. These techniques enable the identification of DNA mutations and/or methylation that characterize different cancers $(19,20)$. However, the fluorimetric technique allows a fast and convenient quantification of the total amount of cfDNA, without distinction between the non-neoplastic and neoplastic fraction. It is plausible that the measurement of the total amount of cfDNA could be a more sensitive biomarker of the neoplastic process because the total amount of cfDNA includes also the fraction derived from apoptosis and necrosis (10-12).

It has been demonstrated that a higher amount of cfDNA is released in the blood and can be isolated from serum and plasma of PCa patients, and that the level of cfDNA in PCa is significantly higher than in patients with BPH (13). Gordian et al. proposed a cut-off of $180 \mathrm{ng} / \mathrm{ml}$ cfDNA to differentiate PCa from BPH patients with PSA levels less than $10 \mathrm{ng} / \mathrm{ml}$ (9) whilst Feng J et al. proposed cfDNA plasma levels of 10 $\mathrm{ng} / \mathrm{ml}$ to differentiate PCa from BPH (13).

Previous studies identified urine cell-free DNA (UCFDNA) integrity as a potentially good marker for the early diagnosis of non-invasive prostate cancer, even though large cohort studies are lacking $(14,15)$. Van Nestle L et al. proposed a urine risk score based on the mRNA liquid biopsy assay (16). Additionally, a urinary three-gene panel (HOXC6, TDRDI and DLX1) has been proposed as a promising tool to identify patients with aggressive $\mathrm{PCa}$, also among those with low PSA values (17).

However, so far, no research has been dedicated to cfDNA assessment in the seminal plasma of $\mathrm{PCa}$ and $\mathrm{BPH}$ patients. cfDNA has been detected in human semen at higher concentrations with respect to other biological fluids $(11,12,18)$. Recently, we reported that the quantification and the electrophoretic characterization of cfDNA in seminal plasma enables the discrimination between $\mathrm{PCa}$ patients and age-matched healthy individuals, demonstrating significantly higher concentrations of scfDNA in the former group, with a distinct electrophoretic pattern $(11,12)$. In the present study this analysis was expanded to include $\mathrm{BPH}$ patients, revealing a significant difference in the concentration levels of cfDNA in $\mathrm{PCa}$ and $\mathrm{BPH}$ patient cohorts (Figure 1). Specifically, a possible cut-off level of $450 \mathrm{ng} / \mathrm{u} \mu \mathrm{l}$ seminal cfDNA is suggested to discriminate between the two distinct groups.

Several methods of cfDNA quantification in serum/plasma have been reported, including qPCR, spectrophotometry, radioimmunoassay and fluorometry. Recently, we compared the standard method of qPCR amplification to the fluorometric assessment by Qubit and we showed high levels of correlation among the two methods (10), confirming that the fluorometry and electrophoresis are both fast, costeffective and reliable procedures to quantify cfDNA and and asses its quality $(11,12)$.

This study has inherent limitations associated with a single institution study. The study has a limited number of participating patients and healthy individuals and requires future studies that will inlcude larger cohorts to confirm the external validity of the study results. Further, seminal fluid cfDNA can only be applied to patients capable of producing a seminal sample.

\section{Conclusion}

Several non-blood biological fluids are potential sources for quantification and characterization of cfDNA in the clinical setting. Among these, seminal fluid is a precious source of nucleic acids, characterized by higher values of cfDNA compared to blood. Seminal fluid, which also contains prostatic secretions, can be adopted as a useful source of biomarkers for differential diagnosis between $\mathrm{PCa}$ and $\mathrm{BPH}$ patients. Seminal fluids of PCa patients were characterized by significantly higher values of cfDNA whilst BPH patients had low seminal cfDNA levels, similar to those observed in healthy volunteers. A cut-off level of $450 \mathrm{ng} / \mu \mathrm{l}$ seminal cfDNA was able to discriminate $\mathrm{PCa}$ from $\mathrm{BPH}$ patients. Seminal cfDNA is a biomarker which can potentially be applied for $\mathrm{PCa}$ risk stratification and therapeutic monitoring.

\section{References}

1 Ferlay J, Soerjomataram I, Dikshit R, Eser S, Mathers C, Rebelo M, Parkin DM, Forman D and Bray F: Cancer incidence and mortality worldwide: sources, methods and major patterns in GLOBOCAN 2012. Int J Cancer 136: E359-386, 2015.

2 Fleischhacker M: Meeting report - First international symposium on circulating nucleic acids in plasma/serum Implications in cancer diagnostics, prognosis or follow up and in prenatal diagnosis - Menthon France, August 18-20, 1999. Eur J Med Res 4: 488-490, 1999.

3 Riethdorf S, Soave A and Rink M: The current status and clinical value of circulating tumor cells and circulating cell-free tumor DNA in bladder cancer. Transl Androl Urol 6: 1090-1110, 2017. 
4 Shankar GM, Balaj L, Stott SL, Nahed B and Carter BS: Liquid biopsy for brain tumors. Expert Rev Mol Diagn 17: 943-947, 2017.

5 Tomasetti M, Amati M, Neuzil J and Santarelli L: Circulating epigenetic biomarkers in lung malignancies: From early diagnosis to therapy. Lung Cancer 107: 65-72, 2017.

6 Kaczor-Urbanowicz KE, Martin Carreras-Presas C, Aro K, Tu M, Garcia-Godoy F and Wong DT: Saliva diagnostics - Current views and directions. Exp Biol Med (Maywood) 242: 459-472, 2017.

7 Bettegowda C, Sausen M, Leary RJ, Kinde I, Wang Y, Agrawal N, Bartlett BR, Wang H, Luber B, Alani RM, Antonarakis ES, Azad NS, Bardelli A, Brem H, Cameron JL, Lee CC, Fecher LA, Gallia GL, Gibbs P, Le D, Giuntoli RL, Goggins M, Hogarty MD, Holdhoff M, Hong S-M, Jiao Y, Juhl HH, Kim JJ, Siravegna G, Laheru DA, Lauricella C, Lim M, Lipson EJ, Marie SKN, Netto GJ, Oliner KS, Olivi A, Olsson L, Riggins GJ, Sartore-Bianchi A, Schmidt K, Shih le-M, Oba-Shinjo SM, Siena S, Theodorescu D, Tie J, Harkins TT, Veronese S, Wang T-L, Weingart JD, Wolfgang CL, Wood LD, Xing D, Hruban RH, Wu J, Allen PJ, Schmidt CM, Choti MA, Velculescu VE, Kinzler KW, Vogelstein B, Papadopoulos $\mathrm{N}$ and Diaz LA: Detection of circulating tumor DNA in early- and late-stage human malignancies. Sci Transl Med 6: 224ra24, 2014.

8 Furuya Y, Fuse H and Masai M: Serum soluble Fas level for detection and staging of prostate cancer. Anticancer Res 21: 3595-3598, 2001

9 Gordian E, Ramachandran K, Reis IM, Manoharan M, Soloway MS and Singal R: Serum free circulating DNA is a useful biomarker to distinguish benign versus malignant prostate disease. Cancer Epidemiol Biomarkers Prev 19: 1984-1991, 2010.

10 Ponti G, Maccaferri M, Manfredini M, Kaleci S, Mandrioli M, Pellacani G, Ozben T, Depenni R, Bianchi G, Pirola GM and Tomasi A: The value of fluorimetry (Qubit) and spectrophotometry (NanoDrop) in the quantification of cell-free DNA (cfDNA) in malignant melanoma and prostate cancer patients. Clin Chim Acta 479: 14-19, 2018.

11 Ponti G, Maccaferri M, Manfredini M, Cotugno M, Pellacani G, Conti A, Micali S, Mandrioli M and Tomasi A: Seminal cell-free DNA molecular profile as a novel diagnostic and prognostic prostate cancer biomarkers. Med Hypotheses 114: 69, 2018.

12 Ponti G, Maccaferri M, Mandrioli M, Manfredini M, Micali S, Cotugno M, Bianchi G, Ozben T, Pellacani G, Del Prete C and Tomasi A: Seminal cell-free DNA assessment as a novel prostate cancer biomarker. Pathol Oncol Res, 2018. doi: 10.1007/s12253-018-0416-6. [Epub ahead of print]
13 Feng J, Gang F, Li X, Jin T, Houbao H, Yu C and Guorong L: Plasma cell-free DNA and its DNA integrity as biomarker to distinguish prostate cancer from benign prostatic hyperplasia in patients with increased serum prostate-specific antigen. Int Urol Nephrol 45: 1023-1028, 2013.

14 Salvi S, Gurioli G, Martignano F, Foca F, Gunelli R, Cicchetti G, De Giorgi U, Zoli W, Calistri D and Casadio V: Urine cellfree DNA integrity analysis for early detection of prostate cancer patients. Dis Markers 2015: 574120, 2015.

15 Casadio V, Calistri D, Salvi S, Gunelli R, Carretta E, Amadori $\mathrm{D}$, Silvestrini R and Zoli W: Urine cell-free DNA integrity as a marker for early prostate cancer diagnosis: a pilot study. BioMed Res Int 2013: 270457, 2013.

16 Neste LV, Hendriks RJ, Dijkstra S, Trooskens G, Cornel EB, Jannink SA, Jong H de, Hessels D, Smit FP, Melchers WJG, Leyten GHJM, Reijke TM de, Vergunst H, Kil P, Knipscheer BC, Kaa CAH de, Mulders PFA, Oort IM van, Criekinge WV and Schalken JA: Detection of high-grade prostate cancer using a urinary molecular biomarker-based risk score. Eur Urol 70: 740-748, 2016.

17 Leyten GHJM, Hessels D, Smit FP, Jannink SA, de Jong H, Melchers WJG, Cornel EB, de Reijke TM, Vergunst H, Kil P, Knipscheer BC, Hulsbergen-van de Kaa CA, Mulders PFA, van Oort IM and Schalken JA: Identification of a candidate gene panel for the early diagnosis of prostate cancer. Clin Cancer Res 21: 3061-3070, 2015.

18 Li HG, Huang SY, Zhou H, Liao AH and Xiong CL: Quick recovery and characterization of cell-free DNA in seminal plasma of normozoospermia and azoospermia: implications for non-invasive genetic utilities. Asian J Androl 11: 703-709, 2009.

19 Komiya K, Nakashima C, Nakamura T, Hirakawa H, Abe T, Ogusu S, Takahashi K, Takeda Y, Egashira Y, Kimura S and Sueoka-Aragane N: Current status and problems of T790M detection, a molecular biomarker of acquired resistance to EGFR tyrosine kinase inhibitors, with liquid biopsy and re-biopsy. Anticancer Res 38: 3559-3566, 2018.

20 Hauser S, Zahalka T, Fechner G, Müller SC and Ellinger J: Serum DNA hypermethylation in patients with kidney cancer: results of a prospective study. Anticancer Res 33: 4651-4656, 2013.
Received July 30, 2018

Revised August 16, 2018

Accepted August 19, 2018 